\title{
Automatic Estimation of the Noise Level Function for Adaptive Blind Denoising
}

\author{
Camille Sutour, \\ Institut für Numerische und Angewandte Mathematik, \\ Westfälische Wilhelms-Universität (WWU) Münster \\ Einsteinstraße 62, 48149 Münster, Germany \\ Email: camille.sutour@uni-muenster.de
}

\author{
Jean-François Aujol and Charles-Alban Deledalle \\ Institut de mathématiques de Bordeaux, CNRS UMR 5251, \\ Bordeaux INP, Université de Bordeaux \\ 351 cours de la Libération, 33405 Talence, France \\ Email: \{jaujol,cdeledal\}@ math.u-bordeaux.fr
}

\begin{abstract}
Image denoising is a fundamental problem in image processing and many powerful algorithms have been developed. However, they often rely on the knowledge of the noise distribution and its parameters. We propose a fully blind denoising method that first estimates the noise level function then uses this estimation for automatic denoising. First we perform the nonparametric detection of homogeneous image regions in order to compute a scatterplot of the noise statistics, then we estimate the noise level function with the least absolute deviation estimator. The noise level function parameters are then directly re-injected into an adaptive denoising algorithm based on the non-local means with no prior model fitting. Results show the performance of the noise estimation and denoising methods, and we provide a robust blind denoising tool.
\end{abstract}

\section{INTRODUCTION}

Image denoising is widely studied in image processing. Many powerful algorithms have been developed recently and achieve outstanding results [1], [2]. However, they often rely on the knowledge of the noise distribution and the noise level, that are in most cases assumed to be known. We propose a blind denoising algorithm that automatically estimates the noise level function, i.e. the function of the noise variance with respect to the image intensities, then re-injects the estimation into a denoising algorithm without any model fitting.

Section II is dedicated to the automatic estimation of spatially uncorrelated, signal-dependent noise from a single image. Variance stabilizing transforms can reduce the dependency between the signal intensity and the noise [3]. Separation techniques have also been extended to specific signal-dependent models, e.g., using a wavelet transform for a Poisson-Gaussian model [4] or using a Gaussian mixture model of patches for additive noise with polynomial variance [5]. The noise can also be distinguished from the signal components by principal component analysis [6] or by selecting blocks with lowest variance [7].

The approach that we follow here [8] relies on the fact that natural images contain homogeneous areas, where the signal to noise ratio is very weak, so only the statistics of the noise intervene. While classic detectors require assumptions on the noise statistics [9], [10], we propose a non-parametric detection of homogeneous areas based on Kendall's rank correlation coefficient [11] that only requires the noise to be spatially uncorrelated. Then we estimate the noise level function (NLF), i.e., the function of the noise variance with respect to the image intensities, as a second order polynomial minimizing the $\ell^{1}$ error on the statistics of these regions.

Then in section III, we use the estimated noise level function for blind denoising. We adapt an adaptive denoising algorithm [12] that performs fast image denoising and is flexible for different noise statistics. The proposed method relies only on the estimated noise level function: the noise is approximated by additive noise with polynomial variance and the denoising algorithm is adapted accordingly.

In section IV, experiments and numerical results show the validity of the proposed estimation and denoising methods, as well as comparisons to the state-of-the-art. We also provide a Matlab implementation for the automatic noise estimation and its application to image and video denoising, that is available for download at https://github.com/csutour/RNLF.

\section{NOISE ESTIMATION}

In this problem, we assume that the observed image $g \in$ $\mathbb{R}^{N}$, where $N$ is the number of pixels of the image, is an observation of a clean unknown image $g^{0}$, corrupted by a spatially uncorrelated signal dependent noise. Hence, $g$ can be modeled as the realization of a random vector $G$ such that $\mathbb{E}[G]=g^{0}$, and

$$
\operatorname{Cov}[G]=\left(\begin{array}{cccc}
\operatorname{NLF}\left(g_{1}^{0}\right) & & & 0 \\
& \operatorname{NLF}\left(g_{2}^{0}\right) & & \\
& & \ddots & \\
0 & & & \operatorname{NLF}\left(g_{N}^{0}\right)
\end{array}\right),
$$

where NLF : $\mathbb{R} \rightarrow \mathbb{R}^{+}$is coined the noise level function. This model hence encompasses spatially uncorrelated, signal dependent noise.

In order to estimate the unknown noise level function, we rely on the fact that most natural images contain homogeneous regions, i.e., areas where the underlying clean signal can be assumed to be constant. In those regions, according to eq. (1), the empirical expectation and variance should provide a punctual estimation of the noise level function. Hence, we seek to detect homogeneous regions with no access to the true underlying signal $g^{0}$ in order to get punctual estimations of the noise level function. Then the NLF can be estimating by fitting a second order polynomial function to the scatterplot. 
a) Noisy image

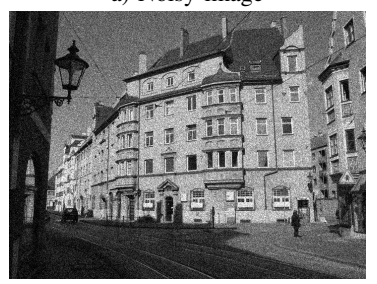

b) $p$-value

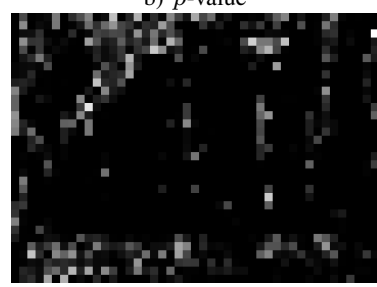

c) Detection

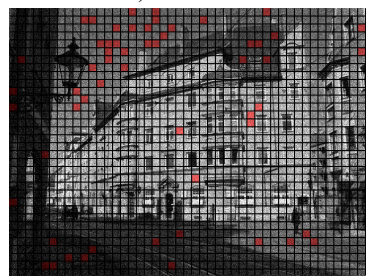

d) Estimation

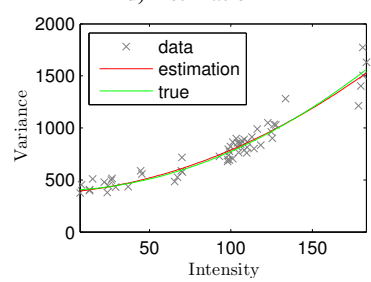

Figure 1. Detection of homogeneous areas in an image corrupted with hybrid noise as the sum of Gaussian, Poisson and multiplicative gamma noise whose NLF parameters are $(a, b, c)=(0.0312,0.75,400)$, resulting in an initial PSNR of 17.93dB. a) Noisy image (range [0, 255]), b) $p$-value (range [black $=0$, white $=1]$ ) of the associated Kendall's $\tau$ coefficient computed within blocks of size $W=16 \times 16$, and c) selected homogeneous blocks (red) by thresholding the $p$-value to reach a probability of detection of $P_{\mathrm{D}}=1-P_{\mathrm{FA}}=0.7$, d) Estimation of the noise level function with the LAD estimator.

\section{A. Detection of homogeneous areas}

The goal is to develop a method that automatically selects homogeneous regions in the image. It is important for such technique not to make any assumption on the nature of the noise. We therefore consider a non-parametric approach whose statistical answer is independent of the noise model. The key idea is that we focus mainly on the rank (i.e. on the relative order) of the pixel values rather than on the values themselves. If the ranking of the pixel values is uniformly random or spatially uncorrelated, then this means that there is no apparent structure in the considered zone.

1) Kendall's $\tau$ coefficient: To measure the correlation of the ranking, we rely on the Kendall's $\tau$ coefficient. Kendall's $\tau$ coefficient is a rank correlation measure [11] that provides a non-parametric hypothesis test for statistical dependence. Let $\left(x_{1}, \cdots, x_{n}\right)$ and $\left(y_{1}, \cdots, y_{n}\right)$ be two sequences of $n$ observations of random variables $X$ and $Y$.

Definition. Kendall's $\tau \in[-1,1]$ coefficient is defined as:

$$
\tau=\frac{1}{n(n-1)} \sum_{1 \leq i, j \leq n} \operatorname{sign}\left(x_{i}-x_{j}\right) \operatorname{sign}\left(y_{i}-y_{j}\right),
$$

assuming that, for all $i \neq j, x_{i} \neq x_{j}$ and $y_{i} \neq y_{j}$. A value $\tau=0$ indicates the absence of correlation between $X$ and $Y$. Distribution of $\tau$. Under the null hypothesis of independence of $X$ and $Y$, the sampling distribution of $\tau$ has an expected value of 0 . In case of large samples, it is approximated by the normal distribution [13]:

$$
\tau \sim \mathcal{N}\left(0, \frac{2(2 n+5)}{9 n(n-1)}\right) .
$$

In fact, it can be used for non-parametric tests as its distribution does not rely on any assumptions regarding the distribution of $X$ and $Y$.

Determining significance. The above coefficient indicates whether the variables are likely to be dependent or not, and its significance is based on the score, which is approximately distributed along a standard normal distribution. The detection is performed by computing the associated $p$-value and rejecting the null hypothesis if the $p$-value is smaller than a predetermined significance level $\alpha$, that corresponds to the desired probability of detection.

2) Homogeneous detection: Kendall's rank correlation coefficient is a non-parametric measure that assesses the statistical dependence between two variables, based on their relative order. In the homogeneous detection problem, we need to estimate whether the samples of a block $g^{\omega}$ of the image $g$ are independent and identically distributed, based on the fact that if the area is homogeneous, then the ranking is spatially uniform. To do so, we look at the statistical dependence between pixels of a block $g^{\omega}$ by dividing the block in two disjoint sequences $g_{1}^{\omega}=\left(g_{2 k}^{\omega}\right)$ and $g_{2}^{\omega}=\left(g_{2 k+1}^{\omega}\right)$ where $g_{2 k}^{\omega}$ and $g_{2 k+1}^{\omega}$ represent neighbor pixel values for a given scan path. If these two variables are found to be independent, this means that there is no relationship between the pixels of the blocks and their neighbors, so we can assume that there is no structure and all fluctuations are only due to noise.

We run $K=4$ tests for horizontal, vertical and the two diagonal neighbors and aggregate them to obtain a more selective estimator. We consider the block to be homogeneous if the test of independence for each direction is satisfied, i.e. if each of the $K$ obtained $p$-values $p_{k}$ reaches a given level of significance $\alpha$. By doing so, the overall level of detection $\alpha_{e q}$ after aggregation is no longer $\alpha$ but smaller and given by

$$
\alpha_{e q}=P\left(\bigcap_{k=1}^{K}\left\{p_{k}>\alpha\right\}\right) .
$$

In order to control the overall level of detection $\alpha_{e q}$, we empirically estimated offline the relation between $\alpha_{e q}$ and $\alpha$.

\section{B. Model estimation}

Once the mean/variance couples $\left(m, s^{2}\right)$ on uniform regions are computed, a model that fits the observed NLF can be estimated. The goal is to find the polynomial coefficients $(a, b, c)$ such that the vector of each estimated variance $s^{2}$ can be represented as $a m^{2}+b m+c$, where $m$ contains the estimated means. To do so, we use the least absolute deviation estimator that minimizes a $L^{1}$-norm, that is known to be more robust to outliers (that might happen due to false homogeneous detection) than the $L^{2}$-norm. The problem is formulated as follows:

$$
\begin{aligned}
\widehat{(a, b, c)} & =\underset{a, b, c}{\operatorname{argmin}}\left\|a m^{2}+b m+c-s^{2}\right\|_{1} \\
& =\underset{a, b, c}{\operatorname{argmin}}\left\|N L F_{(a, b, c)}(m)-s^{2}\right\|_{1} .
\end{aligned}
$$

We can derive an iterative solution, using the preconditioned primal-dual algorithm of Chambolle-Pock [14]. 

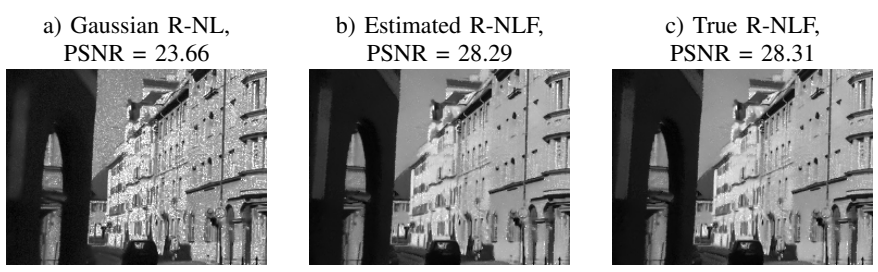

Figure 2. Denoising of a hybrid noise with true parameters $(a, b, c)=$ $(0.0312,0.625,100)$, initial PSNR $=20.34 \mathrm{~dB}$. The noisy image is displayed on Fig. 1-a. a) Standard R-NL assuming Gaussian noise, b) R-NLF with the estimated NLF and c) R-NLF with the true NLF.

\section{Denoising}

Once the noise level function has been estimated, it can be injected into the denoising process, based on the R-NL denoising algorithm [12]. This flexible algorithm allows efficient denoising using solely the noise level function estimation.

\section{A. R-NL: adaptive denoising algorithm}

In previous work [12], we have combined the assumptions of regularity and redundancy provided respectively by the variational methods [15] and the non-local means [16].

1) NL-means: The non-local means algorithm is based on the hypothesis of redundancy of structures inside natural images. It performs a weighted average of pixels with similar neighborhoods. For each pixel $i$ in the image domain $\Omega$, the solution of the NL-means is:

$$
u_{i}^{\mathrm{NL}}=\sum_{j \in \Omega} w_{i, j} g_{j},
$$

where the weights $w_{i, j} \in[0,1]$ select pixels $j$ whose surrounding patch $\rho_{j}$ is similar to the patch $\rho_{i}$ extracted around the central pixel $i$ :

$$
w_{i, j}=\frac{1}{Z_{i}} \exp \left(-\frac{\left|d\left(g^{\rho_{i}}, g^{\rho_{j}}\right)-m_{d}^{\rho}\right|}{s_{d}^{\rho}}\right) .
$$

$Z_{i}$ is a normalization factor and $d$ is a similarity function that evaluates the similarity between patches according to the noise distribution [17], while $m_{d}^{\rho}$ and $s_{d}^{\rho}$ are respectively the mean and standard deviation of the dissimilarity $d$, evaluated empirically on identically distributed noisy patches of size $|\rho|$.

If the NL-means offer an overall good performance, they suffer from two opposite drawbacks: on the one hand they might over-smooth low-contrasted areas due to the selection of irrelevant candidates, while on the other hand they leave a residual noise around edges and singular structures due to the lack of redundancy. These two flaws are respectively referred to as the jittering effect and the rare patch effect.

2) Adaptive regularization of the NL-means: In previous work [12], we reduce these drawbacks in two steps.

Dejittering step: The jittering is due to an over-important variance reduction that produces bias [18]. The proposed method balances the bias-variance compromise by re-injecting noisy data when denoising is irrelevant, i.e. when the variance reduction is too high. We perform an adaptive convex combination between the NL-means solution $u^{\mathrm{NL}}$ and the noisy image $g$ for each each pixel $i$ by:



\section{NLF estimation step}

for each block $g^{\omega}$ do

for each direction $k=1 . . K$ do

Compute $\tau\left(g_{1}^{\omega}, g_{2}^{\omega}\right)$

Compute the $p$-value $p_{k}^{\omega}$

end for

if $\bigcap_{k=1}^{K}\left\{p_{k}^{\omega}>\alpha\right\}$ then

Insert $\left(\operatorname{mean}\left(g^{\omega}\right), \operatorname{Var}\left(g^{\omega}\right)\right)$ to $\left(m, s^{2}\right)$

end if

end for

Estimate $\widehat{(a, b, c)}=\underset{a, b, c}{\operatorname{argmin}}\left\|N L F_{(a, b, c)}(m)-s^{2}\right\|_{1}$.

for $i \in \Omega$ do

NL-means step

Compute $w_{i, j} \leftarrow \frac{1}{Z_{i}} \exp \left(-\frac{\left|d\left(g^{\rho_{i}}, g^{\rho_{j}}\right)-m_{d}^{\rho}\right|}{s_{d}^{\rho}}\right), \forall j \in \mathcal{N}_{i}$

Compute $u_{i}^{\mathrm{NL}} \leftarrow \sum_{j} w_{i, j} g_{j}$

Compute $\left(\hat{\sigma}_{i}^{\mathrm{NL}}\right)^{2} \leftarrow \sum_{j} w_{i, j} g_{j}^{2}-\left(u_{i}^{\mathrm{NL}}\right)^{2}$

Compute $\left(\sigma_{i}^{\text {noise }}\right)^{2}=a\left(u_{i}^{\mathrm{NL}}\right)^{2}+b\left(u_{i}^{\mathrm{NL}}\right)+c$

Dejittering step

Compute $\alpha_{i} \leftarrow \frac{\left|\left(\hat{\sigma}_{i}^{\mathrm{NL}}\right)^{2}-\left(\sigma_{i}^{\text {noise }}\right)^{2}\right|}{\left|\left(\hat{\sigma}_{i}^{\mathrm{NL}}\right)^{2}-\left(\sigma_{i}^{\text {noise }}\right)^{2}\right|+\left(\sigma_{i}^{\text {noise }}\right)^{2}}$

Update $u_{i}^{\mathrm{NL}} \leftarrow\left(1-\alpha_{i}\right) u_{i}^{\mathrm{NL}}+\alpha_{i} g_{i}$

Update $w_{i, j} \leftarrow\left(1-\alpha_{i}\right) w_{i, j}+\alpha_{i} \delta_{i, j}$

Compute $\lambda_{i} \leftarrow \gamma\left(\sum_{j} w_{i, j}^{2}\right)$

end for

\section{Minimization step}

$$
\begin{aligned}
& u^{\mathrm{R}-\mathrm{NLF}}=\underset{u}{\operatorname{argmin}} \sum_{i \in \Omega} \lambda_{i} \frac{\left(u_{i}-u_{i}^{\mathrm{NL}}\right)^{2}}{2 \mathrm{NLF}_{(a, b, c)}\left(u_{i}^{\mathrm{NL}}\right)}+\mathrm{TV}(u) \\
& \text { return } u^{\mathrm{R}-\mathrm{NLF}}
\end{aligned}
$$

$$
u_{i}^{\mathrm{NLDJ}}=\left(1-\alpha_{i}\right) u_{i}^{\mathrm{NL}}+\alpha_{i} g_{i}=\sum_{j \in \Omega} w_{i, j}^{\mathrm{NLDJ}} g_{j},
$$

where the weights $w_{i, j}^{\mathrm{NLDJ}}=\left(1-\alpha_{i}\right) w_{i, j}^{\mathrm{NL}}+\alpha_{i} \delta_{i, j}\left(\delta_{i, j}\right.$ is Kronecker's symbol) are in fact a readjustment of the initial weights $w_{i, j}^{\mathrm{NL}}$, and $\alpha_{i}$ is a jittering index given by:

$$
\alpha_{i}=\frac{\left|\left(\hat{\sigma}_{i}^{\mathrm{NL}}\right)^{2}-\left(\sigma_{i}^{\text {noise }}\right)^{2}\right|}{\left|\left(\hat{\sigma}_{i}^{\mathrm{NL}}\right)^{2}-\left(\sigma_{i}^{\text {noise }}\right)^{2}\right|+\left(\sigma_{i}^{\text {noise }}\right)^{2}} .
$$

$\left(\sigma_{i}^{\text {noise }}\right)^{2}$ refers to the noise variance, and $\left(\hat{\sigma}_{i}^{\mathrm{NL}}\right)^{2}$ is the non local variance that reflects the variance of the selected candidates in the weighted average. Besides, the residual variance at pixel $i$ of the dejittered solution $u^{\mathrm{NLDJ}}$ is given by:

$$
\left(\hat{\sigma}_{i}^{\text {residual }}\right)^{2}=\left[\sum_{j \in \Omega}\left(w_{i, j}^{\mathrm{NLJ}}\right)^{2}\right]\left(\sigma_{i}^{\mathrm{noise}}\right)^{2} .
$$


The quantity $\sum_{j \in \Omega}\left(w_{i, j}^{\mathrm{NLDJ}}\right)^{2}$ reflects the amount of noise that has been removed from pixel $i$, providing a performance index. Regularization step: The performance index $\left(\hat{\sigma}_{i}^{\text {residual }}\right)^{2}$ is then used to reduce the rare patch effect, through an adaptive regularization based on a non-local data fidelity term and a total variation (TV) regularization [15]:

$$
\begin{aligned}
u^{\mathrm{R}-\mathrm{NL}} & =\underset{u \in \mathbb{R}^{N}}{\operatorname{argmin}} \sum_{i \in \Omega} \lambda_{i} \sum_{j \in \Omega} w_{i, j}\left(g_{j}-u_{i}\right)^{2}+\mathrm{TV}(u) \\
& =\underset{u \in \mathbb{R}^{N}}{\operatorname{argmin}} \sum_{i \in \Omega} \lambda_{i}\left(u_{i}-u_{i}^{\mathrm{NL}}\right)^{2}+\mathrm{TV}(u),
\end{aligned}
$$

where $\operatorname{TV}(u)=\sum_{i \in \Omega}\left\|(\nabla u)_{i}\right\|$, and $\lambda_{i}$ is an adaptive regularization parameter given by:

$$
\lambda_{i}=\gamma\left(\frac{\hat{\sigma}_{i}^{\text {residual }}}{\sigma_{i}^{\text {noise }}}\right)^{-1}=\gamma\left(\sum_{j \in \Omega} w_{i, j}^{2}\right)^{-1 / 2} .
$$

\section{B. R-NLF: blind denoising}

Thanks to the good properties of the non-local means and the variational methods, R-NL can readily be adopted to different noise models, by adapting the similarity measure between patches according to the noise statistics [17], as well as the data fidelity term in the regularization process [12].

For blind denoising, we do not estimate a given model, going through hypothesis tests, but we rather use directly the estimated NLF parameters. For this purpose, we approximate the noise by additive, signal-dependent Gaussian noise, with second order polynomial variance, such that the noisy image $g$ is a realization of the random variable $G$ given by:

$$
G=f+\operatorname{NLF}_{(a, b, c)}(f) \cdot \varepsilon,
$$

with $\operatorname{NLF}_{(a, b, c)}(f)=a f^{2}+b f+c$ and $\varepsilon \sim \mathcal{N}(0,1)$.

Then the R-NLF algorithm is derived from R-NL, taking into account the signal dependence without direct knowledge of the noise distribution, but only of the $(\widehat{a, b, c})$ parameters of the estimated NLF. The dissimilarity measure $d$ is then adapted as follows:

$$
d\left(g^{\rho_{i}}, g^{\rho_{j}}\right)=\frac{1}{|\rho|} \sum_{k=1}^{|\rho|} \frac{\left(g_{k}^{\rho_{i}}-g_{k}^{\rho_{j}}\right)^{2}}{\operatorname{NLF}_{\widehat{(a, b, c)}}\left(g_{k}^{\rho_{i}}\right)+\mathrm{NLF}_{(\widehat{a, b, c})}\left(g_{k}^{\rho_{j}}\right)} .
$$

The dejittering step is straightforward; it relies on the computation of the index $\alpha_{i}$, based on the non local variance $\left(\widehat{\sigma}_{i}^{\mathrm{NL}}\right)^{2}$ and the noise variance $\left(\sigma_{i}^{\text {noise }}\right)^{2}$, computed as follows:

$$
\left(\sigma_{i}^{\text {noise }}\right)^{2}=\mathrm{NLF}_{(\widehat{a, b, c})}\left(u_{i}^{\mathrm{NL}}\right)=\hat{a}\left(u_{i}^{\mathrm{NL}}\right)^{2}+\hat{b}\left(u_{i}^{\mathrm{NL}}\right)+\hat{c} .
$$

Finally, using the polynomial variance Gaussian model, problem (11) becomes:

$$
u^{\mathrm{R}-\mathrm{NLF}}=\underset{u \in \mathbb{R}^{N}}{\operatorname{argmin}} \sum_{i \in \Omega} \lambda_{i} \frac{\left(u_{i}-u_{i}^{\mathrm{NL}}\right)^{2}}{2 \mathrm{NLF}_{(\widehat{a, b, c})}\left(u_{i}^{\mathrm{NL}}\right)}+\mathrm{TV}(u) .
$$

Similarly to the Gaussian case, this minimization problem is then solved using the primal-dual algorithm [14]. The whole blind denoising process is summarized in Algorithm 1.
Table I

MEAN RELATIVE ERROR (MRE) FOR POISSON-GAUSSIAN AND HYBRID NOISE WiTh THE GAUSSIAN-CAUCHY MIXTURE MODEL [4], THE PCA METHOd [6], THE PERCENTILE METHOd [7], Noise Clinic [19], [20], THE VST BASED METHOD [3] (ONLY AFFINE MODEL) AND OUR

ALGORITHM (AFFINE OR SECOND ORDER MODEL), AND PSNR AFTER DENOISING WITH THE R-NLF ALGORITHM, USING THE ESTIMATED NLF.

\begin{tabular}{ccccccc}
\hline & \multicolumn{2}{c}{ Affine noise } & \multicolumn{2}{c}{ Affine noise } & \multicolumn{2}{c}{ Hybrid noise } \\
\hline Estimator & $M R E$ & $P S N R$ & $M R E$ & $P S N R$ & $M R E$ & $P S N R$ \\
\hline Gaussian-Cauchy [4] & 0.093 & 29.051 & 0.045 & 26.318 & 0.051 & 26.810 \\
PCA [6] & 0.219 & 28.324 & 0.873 & 24.127 & 0.454 & 23.923 \\
Percentile [7] & 0.084 & 28.994 & 0.117 & 26.072 & 0.148 & 26.057 \\
Noise Clinic [19] & 0.327 & 27.616 & 0.373 & 24.267 & 0.403 & 24.201 \\
[20] & $\backslash$ & 28.114 & $\backslash$ & 25.009 & $\backslash$ & 25.509 \\
VST [3] & 0.040 & 29.124 & 0.035 & 26.361 & $\backslash$ & $\backslash$ \\
Prop. affine & 0.078 & 29.062 & 0.057 & 26.308 & $\backslash$ & $\backslash$ \\
Prop. hybrid & 0.080 & 28.946 & 0.059 & 26.115 & 0.070 & 26.628 \\
R-NLF (real) & $\backslash$ & 29.159 & $\backslash$ & 26.429 & $\backslash$ & 26.766 \\
\hline
\end{tabular}
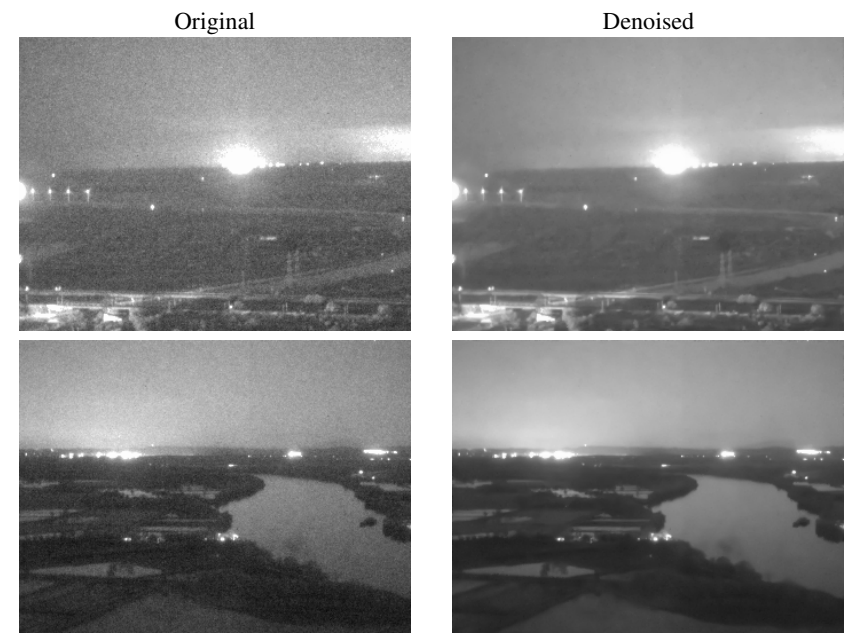

Figure 3. Blind denoising of night vision images acquired from an helicopter using a light intensifier coupled to a CCD camera.

\section{EXPERIMENTS AND RESULTS}

In this section, we discuss and compare the efficiency of the proposed approach with regards to the noise estimation and the blind image denoising. For the sake of replicability, a Matlab implementation for the automatic noise estimation and its application to image and video denoising is available for download at https://github.com/csutour/RNLF.

Figure 1 illustrates the noise estimation process, and Fig. 2 shows the denoising results of an image corrupted with simulated hybrid noise. On Fig. 2-a, the noise is assumed to be Gaussian, so the result suffers from some artifacts due to the fact that the noise variance should not be assumed to be constant over the whole image. On Fig. 2-b, the polynomial NLF is estimated and plugged into the denoising process while on Fig. 2-c, the real noise parameters are used. The similar results show the reliability of the estimation.

\section{A. Comparison to state of the art}

We validate the proposed approach with respect to the state of the art algorithms that perform noise estimation and/or blind denoising. Based on the database of 150 natural images ${ }^{1}$, we generate a set of noisy images, either with Poisson-Gaussian

\footnotetext{
${ }^{1}$ http://www.gipsa-lab.grenoble-inp.fr/ laurent.condat/imagebase.html
} 
noise with low or high noise level or with a mixture of Gaussian, Poisson and Gamma noise. We estimate the noise parameters with the different estimators: the Gaussian-Cauchy mixture model [4] which is the most general model, the PCA method [6], the percentile method [7], and the Noise Clinic estimation [19], that estimate frequency-dependent noise but that we use here for the estimation of affine or hybrid noise, the estimation based on the variance stabilization transform (VST) [3] that applies only for Poisson-Gaussian noise, and our algorithm that can estimate either a given model (e.g., affine) or a general second order one. Based on the knowledge of the real noise parameters $(a, b, c)$, we compute the mean relative error

$$
\operatorname{MRE}(\widehat{a, b, c})=\frac{1}{|I|} \sum_{f \in I} \frac{\left|\mathrm{NLF}_{(a, b, c)}(f)-\mathrm{NLF}_{(\widehat{a, b, c})}(f)\right|}{\operatorname{NLF}_{(a, b, c)}(f)},
$$

where $I$ is a discretization of the interval of image intensities. The level of detection $\alpha$ as well as the block size $W$ have also been empirically optimized using this mean relative error.

Then we plug the estimated NLF parameters for each method into the R-NLF algorithm, and we compute the obtained PSNR. We also compare the denoising results to the Noise-Clinic denoising algorithm [20] and to the results of the R-NLF denoising algorithm using the true noise parameters (so there is no noise estimation error in these cases). Table I illustrates the estimation and denoising performance of the suitable estimators for Poisson-Gaussian and hybrid noise. Results show that our estimation method offers comparable results to the Gaussian-Cauchy method, and that reliable noise estimations offer good denoising performance.

\section{B. Night vision application}

The proposed blind denoising algorithm has been used on night vision images. In order to improve night vision for helicopter pilots, a light intensifier tube multiplies the number of photons in order to artificially increase light, then the output is coupled to a CCD (Charge Coupled Device) camera and the images are projected onto the helmet's visor in order to provide a head-up display. However, the obtained images suffer from heavy non-Gaussian noise. Using the blind denoising algorithm, we can first estimate the unknown noise level function then apply the adaptive denoising algorithm. Results are displayed on Fig. 3.

\section{Conclusion}

We have developed a fully automatic blind denoising method that relies on the estimation of the noise level function and robust image denoising. The noise estimation is performed using the non-parametric detection of homogeneous regions based on Kendall's $\tau$ coefficient between neighbors, then the noise level function is estimated thanks to a $L^{1}$-minimization. Then the noise level function is directly re-injected into a robust denoising algorithm based on an adaptive regularization of the non-local means. This method can encompass a general second order noise model, and results on synthetic images show show the performance of both the noise estimation and the denoising process. Furthermore, we provide a Matlab implementation for an easy access to the developed tools. Future work might lead to the study of a more general noise model, that could also encompass spatially varying noise level functions and spatially correlated noise.

\section{ACKNOWLEDGMENT}

C. Sutour thanks the DGA and the Aquitaine region for funding her PhD. J.-F. Aujol acknowledges the support of the Institut Universitaire de France. This study has been carried out with financial support from the French State, managed by the French National Research Agency (ANR) in the frame of the "Investments for the future" Programme IdEx Bordeaux - CPU (ANR-10-IDEX-03-02).

\section{REFERENCES}

[1] M. Elad and M. Aharon, "Image denoising via sparse and redundant representations over learned dictionaries," IEEE Trans. Image Process., vol. 15(12):3736-3745, 2006.

[2] K. Dabov, A. Foi, V. Katkovnik, and K. Egiazarian, "Image denoising by sparse 3D transform-domain collaborative filtering," IEEE Trans. Image Process., vol. 16(8):2080-2095, 2007.

[3] S. Pyatykh and J. Hesser, "Image sensor noise parameter estimation by variance stabilization and normality assessment," IEEE Trans. Image Process., vol. 23(9):3990-3998, 2014.

[4] L. Azzari and A. Foi, "Gaussian-cauchy mixture modeling for robust signal-dependent noise estimation," in IEEE Int. Conf. Acoustics, Speech, and Signal Processing (ICASSP), 2014, pp. 5357-5361.

[5] _ , "Indirect estimation of signal-dependent noise with nonadaptive heterogeneous samples." IEEE Trans. Image Process., vol. 23(8):34-59, 2014.

[6] M. Colom and A. Buades, "Analysis and extension of the pca method, estimating a noise curve from a single image," IPOL, 2014.

[7] — , "Analysis and extension of the percentile method, estimating a noise curve from a single image," IPOL, vol. 3:332-359, 2013.

[8] C. Sutour, C.-A. Deledalle, and J.-F. Aujol, "Estimation of the noise level function based on a non-parametric detection of homogeneous image regions," SIAM Journal Imaging Sci., vol. 8(4):2622-2661, 2015.

[9] K. Chehdi and M. Sabri, "A new approach to identify the nature of the noise affecting an image," in IEEE Int. Conf. Acoustics, Speech, and Signal Processing (ICASSP), vol. 3:285-288, 1992.

[10] L. Beaurepaire, K. Chehdi, and B. Vozel, "Identification of the nature of noise and estimation of its statistical parameters by analysis of local histograms," in IEEE Int. Conf. Acoustics, Speech, and Signal Processing (ICASSP), vol. 4:2805-2808, 1997.

[11] M. Kendall, "A new measure of rank correlation," Biometrika, vol. 30(12):81-93, 1938 .

[12] C. Sutour, C.-A. Deledalle, and J.-F. Aujol, "Adaptive regularization of the NL-means: Application to image and video denoising," IEEE Trans. Image Process., vol. 23(8):3506-3521, 2014.

[13] A. Prokhorov, "Kendall coefficient of rank correlation," Online Encyclopedia of Mathematics, 2001.

[14] A. Chambolle and T. Pock, "A first-order primal-dual algorithm for convex problems with applications to imaging," Journal of Mathematical Imaging and Vision, vol. 40(1):120-145, 2011.

[15] L. Rudin, S. Osher, and E. Fatemi, "Nonlinear total variation based noise removal algorithms," Physica D, vol. 60(1):259-268, 1992.

[16] A. Buades, B. Coll, and J.-M. Morel, "A review of image denoising algorithms, with a new one," SIAM Journal Multiscale Model. Simul., vol. 4(2):490-53, 2005.

[17] C.-A. Deledalle, L. Denis, and F. Tupin, "How to compare noisy patches? Patch similarity beyond Gaussian noise," International Journal of Computer Vision, vol. 99(1):86-102, 2012.

[18] C. Kervrann, J. Boulanger, and P. Coupé, "Bayesian non-local means filter, image redundancy and adaptive dictionaries for noise removal," in SSVM. Springer, 2007, pp. 520-532.

[19] M. Colom, M. Lebrun, A. Buades, and J.-M. Morel, "A non-parametric approach for the estimation of intensity-frequency dependent noise," in IEEE Int. Conf. Image Process. (ICIP), 2014, pp. 4261-4265.

[20] M. Lebrun, M. Colom, and J.-M. Morel, "The noise-clinic: a universal blind denoising algorithm," in IEEE Int. Conf. Image Process. (ICIP), 2014, pp. 2674-2678. 